\title{
Short-time decoherence of Josephson charge qubits
}

\author{
Xian-Ting Liang \\ Department of Physics and Institute of Modern Physics, Ningbo University, Ningbo 315211, China \\ Abstract \\ In this paper we investigate the short-time decoherence of single Josephson charge qubit (JCQ). The measure of decoherence \\ is chosen as the maximum norm of the deviation density operator. It is shown that when the temperature low enough (for \\ example $T=30 \mathrm{mK}$ ), within the elementary gate-operation time $\tau^{g} \sim 12.7 \mathrm{ps}$, the decoherence is smaller than $10^{-4}$ at present \\ setup of JCQ. The Josephson charge qubit is suitable to take the blocks for quantum computations according to the DiVincenzo
} low decoherence criterion.

PACS numbers: 03.65.Ta, 03.65.Yz, 85.25.Cp

Keywords: Decoherence, Josephson junction, Quantum computation

\section{INTRODUCTION}

David DiVincenzo put forward five criteria for the candidates of quantum computing hardware to be satisfied 1]. One of which is the low decoherence. An approximate benchmark of the criterion is a fidelity loss no more than $\sim 10^{-4}$ per elementary quantum gate operation. The Josephson junction is considered to be a promising physical realization of qubit. So it is a very significative work to investigate the decoherence of the qubits based on the Josephson junctions. To perform quantitative studying of the decoherence for a given system, in general, one must firstly solve the quantum dynamics problem of the system coupled to the environment. Many kinds of methods have been used for this purposes [2] [3]. The decoherence of the qubit based on Josephson junctions have been studied by many authors. In their investigations the dynamics appeal to the Redfield formalism [4] or Bloch-type [5] master equations, where the Markov approximation are used [6]. The approximation is usually used to evaluate approach to the thermal state at large times. However, a quantum gate operation is finished in a instantaneous time so the approximation may be not suitable to investigate the decoherence of the qubit gates for quantum computing purposes. Recently, V. Privman et al. introduced two measures to quantify the shorttime decoherence 7] 8]. The measures are based on the short-time approximation of the split-operator and derived from a operator norm $\|A\|$ (seeing the following definition). By using these measures V. Privman et al. investigated some spin-boson models. In this paper we use one of the measures investigating the short-time decoherence of the single Josephson charge qubit (JCQ) operations.

\section{JCQ HAMILTONIAN AND A MEASURE OF DECOHERENCE}

In this section we firstly review the JCQ model and then introduce the measure of decoherence used in this paper. The single JCQ Hamiltonian is [5]

$$
H_{R}=E_{c h}\left(n-n_{g}\right)^{2}-E_{J} \cos \varphi
$$

Here, $E_{c h}=e^{2} /\left(C_{g}+C_{J}\right)$ is the charging energy; $E_{J}=$ $I_{c} \hbar / 2 e$ is the Josephson coupling energy [9], where $I_{c}$ is the critical current of the Josephson junction, $\hbar$ the Planck's constant divided $2 \pi$, and $e$ the charge of electron; $n_{g}=C_{g} V_{g} / 2 e$ is the dimensionless gate charge, where $C_{g}$ is the gate capacitance, $V_{g}$ the controllable gate voltage. The number operator $n$ of (excess) Cooper pair on the island, and the phase $\varphi$ of the superconducting order parameter, are quantum mechanically conjugate. Because the Josephson coupling energy $E_{J}$ is much smaller than the charging energy $E_{c h}$, and both of them are much smaller than the superconducting energy gap $\Delta$, the Hamiltonian Eq.(1) can be parameterized by the number of the Cooper pairs $n$ on the island as [5] 10]

$$
\begin{aligned}
H_{R}= & \sum_{n}\left\{E_{c h}\left(n-n_{g}\right)^{2}|n\rangle\langle n|\right. \\
& \left.-\frac{1}{2} E_{J}[|n\rangle\langle n+1|+| n+1\rangle\langle n|]\right\} .
\end{aligned}
$$

When $n_{g}$ is modulated to a half-integer, say $n_{g}=1 / 2$ the levels of two adjacent states are close to each other, the Josephson tunneling mixes them strongly. When the temperature $T$ is low enough the system can be reduced to a two-state system (qubit) because all other charge states have much higher energy and can be neglected. So the Hamiltonian of the system can approximately reads

$$
H_{s}=-\frac{1}{2} B_{z} \sigma_{z}-\frac{1}{2} B_{x} \sigma_{x}
$$

where $B_{z}=E_{c h}\left(1-2 n_{g}\right)$ and $B_{x}=E_{J}$. Eq.(3) is similar to the ideal single qubit model [5], but it can be modulated only by changing one parameter $B_{z}$. However, changing the parameter $B_{z}$ (through switching the gate voltage) one can perform the required one-bit operations. If, for example, one chooses the idle state far to the left from the degeneracy point, the eigenstate lose to $|0\rangle$ and $|1\rangle$. Then switching the system suddenly to 
the degeneracy point for a time $\tau$ and back produces a rotation in spin space [5],

$$
U_{J}=\exp \left(\frac{i E_{J} \tau}{2} \sigma_{x}\right)=\left(\begin{array}{ll}
\cos \frac{\tau E_{J}}{2} & i \sin \frac{\tau E_{J}}{2} \\
i \sin \frac{\tau E_{J}}{2} & \cos \frac{\tau E_{J}}{2}
\end{array}\right) .
$$

This can be obtained by modulating the gate voltage and making $B_{z}=E_{c h}\left(1-2 n_{g}\right)=0$. So it is interesting for our to investigate the decoherence of this kind of qubit. If we consider the interaction of the qubit and its environment the total Hamiltonian becomes

$$
H=H_{s}+H_{I}+H_{B},
$$

where $H_{B}$ is the environment Hamiltonian which is usually modelled by a bath of an infinite number of the harmonic oscillator models which is equivalence to an infinity-mode electromagnetic field. The dissipation and the decoherence of the quantum systems is considered because of the coupling of the quantum system and the fluctuating electromagnetic field. In the JCQ model, the coupling of the qubit with the electromagnetic fluctuations can be modeled by a impedance $Z(\omega)$, placed in series with the voltage (see Fig.1 of Ref.[12]). The bath Hamiltonian $H_{B}$ and the interaction Hamiltonian $H_{I}$ are

$$
H_{B}=\sum_{k} M_{k}, \quad H_{I}=\Lambda_{s} \sum_{k} N_{k}
$$

where $\Lambda_{s}=\sigma_{z}$ and

$$
M_{k}=\omega_{k} a_{k}^{\dagger} a_{k}, N_{k}=g_{k} a_{k}^{\dagger}+g_{k}^{*} a_{k} .
$$

Here, $\omega_{k}$ are the bath mode frequencies, $a_{k}^{\dagger}$ and $a_{k}$ are bosonic create and annihilation operators. $N_{k}$ are the freedom of environment and it is direct proportion to the fluctuations of the voltage of the external circuits [5] 11] and $g_{k}$ are the coupling constants.

Because the bath modes are infinite, their frequencies can be taken continuous. The spectral density of the continuous bath modes is

$$
J_{v}(\omega)=\sum_{k} g_{k}^{2} \delta\left(\omega-\omega_{k}\right)
$$

because it can result in a same Johnson-Nyquist relation which can be obtained from fluctuation-dissipation theorem on this system. On the other hand, at low-frequency, the spectral density behavior is

$$
J_{v}(\omega)=\eta \omega^{s} \exp \left(\omega / \omega_{c}\right),
$$

where $\omega_{c}$ is the high-frequency cut-off and $\eta$ is the dimensionless strength of the dissipation. According to Ref. 12 we know that in the particular interest ohmic case $(s=1)$ [13], for the model of JCQ coupling with electromagnetic fluctuations (see Fig.1 of Ref.[12]),

$$
\eta=\frac{R}{R_{Q}}\left(\frac{C_{t}}{C_{J}}\right)^{2}
$$

where $R_{Q}=(2 e)^{2} / h$ is the (superconducting) resistance quantum. From Eqs. (8) and (9) we know that for a ohmic bath

$$
g_{k}^{2} W(\omega)=\eta \omega \exp \left(\omega / \omega_{c}\right),
$$

where $W(\omega)$ is the density of states.

There are many measures to characterize the decoherence. Usually the environment being assumed to be a large macroscopic the interaction with it leads to the thermal equilibrium at temperature $T$. In this case, Markovian type approximations can be used to quantified the decoherent process and it usually yields the exponential decay of the density matrix elements in the energy basis of the Hamiltonian $H_{s}$. In this time scale the measures of entropy and the first entropy are used for quantifying the decoherence. But the decoherence of the qubit gate operations cannot be characterized by this methods because the time of the elementary quantum gate operation is much shorter than the thermal relaxation time. It has been shown that the norms $\|\sigma\|_{\lambda}$ is useful for describing the decoherence of the short-time evolution. Here $\sigma$ is the deviation operator defined as

$$
\sigma(\tau)=\rho(\tau)-\rho^{i}(\tau),
$$

where $\rho(\tau)$ and $\rho^{i}(\tau)$ are density matrixes of the "real" evolution (with interaction) and the "ideal" one (without interaction) of the investigated system. $\|\sigma\|_{\lambda}$ is defined as

$$
\|\sigma\|_{\lambda}=\sup _{\varphi \neq 0}\left(\frac{\langle\varphi|\sigma| \varphi\rangle}{\langle\varphi \mid \varphi\rangle}\right)^{\frac{1}{2}} .
$$

For a qubit, the norm can be given by

$$
\|\sigma\|_{\lambda}=\sqrt{\left|\sigma_{10}\right|^{2}+\left|\sigma_{11}\right|^{2}} .
$$

For a given system, the norm $\|\sigma\|_{\lambda}$ increase with time, reflecting the decoherence of the system. However, in general it is oscillated at the system's internal frequency. Thus, the decohering effect of the bath is better quantified by the maximal operator norm, $D(t)$. This norm is also defined a measure for characterizing the short-time decoherence.

$$
D(\tau)=\sup _{\rho(0)}\left(\|\sigma(\tau, \rho(0))\|_{\lambda}\right) .
$$

In the following, we will calculate the $\|\sigma\|_{\lambda}$ then the decoherence $D$ of the JCQ.

\section{DECOHERENCE OF JCQ OPERATIONS}

As shown in above subsection, to calculate the decoherence of the JCQ we must study its evolution under the interaction of the qubit with its environment. Suppose the initial state of the system be $R(0)=\rho(0) \otimes \Theta$, where 
$\rho(0)$ is the initial state of JCQ and $\Theta$ is the initial state of the environment, which is the product of the bath modes density matrices $\theta_{k}$. In the initial states, each bath mode $k$ is assumed to be thermalized, namely,

$$
\theta_{k}=\frac{e^{-\beta M_{k}}}{\operatorname{Tr}_{k}\left(e^{-\beta M_{k}}\right)}
$$

where $\beta=1 / k T, k$ is the Boltzmann constant. So the evolution operator may be

$$
U=e^{-i H \tau / \hbar}=e^{-i\left(H_{s}+H_{I}+H_{B}\right) \tau / \hbar} .
$$

In the following we set $t=\tau / \hbar$. Due to non-conservation of $H_{s}$ in this system, the evolution operator cannot be in a general way expressed as $e^{-i H_{s} t} e^{-i\left(H_{I}+H_{B}\right) t}$. But in the sort-time approximation, the operator can be approximately expressed as 14] [16]

$$
U=e^{-i H_{s} t / 2} e^{-i\left(H_{I}+H_{B}\right) t} e^{-i H_{s} t / 2}+o\left(t^{3}\right) .
$$

It has been proved that the expression is accurate enough for the time being short to the characteristic time. So the density matrix elements of the reduced density matrix $\rho(t)$ in the basis of operator $H_{s}$ can be expressed as

$$
\begin{aligned}
\rho_{m n}= & \operatorname{Tr}_{B}\left\langle\varphi_{m}\right| e^{-i H_{s} t / 2} e^{-i\left(H_{I}+H_{B}\right) t} e^{-i H_{s} t / 2} R(0) \\
& e^{i H_{s} t / 2} e^{i\left(H_{I}+H_{B}\right) t} e^{i H_{s} t / 2}\left|\varphi_{n}\right\rangle .
\end{aligned}
$$

By use of the completeness relation $\sum|\cdot\rangle\langle\cdot|=1$, we have

$$
e^{ \pm j H_{s} t / 2}=\sum_{j=0,1} e^{ \pm i t \lambda_{j}}\left|\varphi_{j}\right\rangle\left\langle\varphi_{j}\right|
$$

where

$$
\lambda_{0,1}= \pm \frac{B_{x}}{2}= \pm \frac{E_{J}}{2}
$$

and

$$
\begin{aligned}
& \left|\varphi_{0}\right\rangle=\frac{1}{\sqrt{2}}(|0\rangle-|1\rangle), \\
& \left|\varphi_{1}\right\rangle=\frac{1}{\sqrt{2}}(|0\rangle+|1\rangle) .
\end{aligned}
$$

Similarly,

$$
e^{ \pm i\left(H_{I}+H_{B}\right) t}=\sum_{n=0,1} e^{ \pm i\left(\chi_{n} \sum_{k} J_{k}+\sum_{k} M_{k}\right) t}|n\rangle\langle n|,
$$

where $\chi_{0,1}= \pm 1$ and $|n\rangle=|0\rangle$ or $|1\rangle$, are the eigenvalues and eigenstates of operator $\sigma_{z}$. So we have

$$
\begin{aligned}
\rho_{m n}(t)= & \operatorname{Tr}_{B}\left\langle\varphi_{m}\left|\sum_{\alpha=0,1} e^{-i t \lambda_{\alpha}}\right| \varphi_{\alpha}\right\rangle\left\langle\varphi_{\alpha}\right| \\
& \sum_{\xi=0,1} e^{-i\left(\chi_{\xi} \sum_{k} J_{k}+\sum_{k} M_{k}\right) t}|\xi\rangle\langle\xi| \\
& \sum_{\beta=0,1} e^{-i t \lambda_{\beta}}\left|\varphi_{\beta}\right\rangle\left\langle\varphi_{\beta}\left|\sum_{p, q=0,1}\right| \varphi_{p}\right\rangle\left\langle\varphi_{q}\right| \\
& \rho_{p q}(0) \prod_{k} \theta_{k} \sum_{\mu=0,1} e^{i t \lambda_{\mu}}\left|\varphi_{\mu}\right\rangle\left\langle\varphi_{\mu}\right| \\
& \sum_{\varsigma=0,1} e^{i\left(\chi_{\varsigma} \sum_{k} J_{k}+\sum_{k} M_{k}\right) t}|\varsigma\rangle\langle\varsigma| \\
& \sum_{\nu=0,1} e^{i t \lambda_{\nu}}\left|\varphi_{\nu}\right\rangle\left\langle\varphi_{\nu} \mid \varphi_{n}\right\rangle,
\end{aligned}
$$

namely,

$$
\begin{aligned}
\rho_{m n}(t)= & \sum_{\alpha, \beta, \xi, \varsigma, p, q, \mu, \nu=0,1} e^{i t\left(\lambda_{\mu}+\lambda_{\nu}-\lambda_{\alpha}-\lambda_{\beta}\right)}\left\langle\varphi_{m} \mid \varphi_{\alpha}\right\rangle \\
& \left\langle\varphi_{\alpha} \mid \xi\right\rangle\left\langle\xi \mid \varphi_{\beta}\right\rangle\left\langle\varphi_{\beta} \mid \varphi_{p}\right\rangle\left\langle\varphi_{q} \mid \varphi_{\mu}\right\rangle\left\langle\varphi_{\mu} \mid \varsigma\right\rangle \\
& \left\langle\varsigma \mid \varphi_{\nu}\right\rangle\left\langle\varphi_{\nu} \mid \varphi_{n}\right\rangle \operatorname{Tr}_{B}\left[e^{-i\left(\chi_{\xi} \sum_{k} J_{k}+\sum_{k} M_{k}\right) t}\right. \\
& \left.\rho_{p q}(0) \prod_{k} \theta_{k} e^{i\left(\chi_{\varsigma} \sum_{k} J_{k}+\sum_{k} M_{k}\right) t}\right] .
\end{aligned}
$$

In the Eq. (25) the $\operatorname{Tr}_{B}$ term is same as that in Ref. [7]. Enlightened by Privman's works we can easily obtain

$$
\begin{aligned}
\rho_{m n}(t)= & \sum_{\alpha, \beta, \xi, \varsigma, p, q, \mu, \nu=0,1} e^{i t\left(\lambda_{\mu}+\lambda_{\nu}-\lambda_{\alpha}-\lambda_{\beta}\right)} \\
& \left\langle\varphi_{m} \mid \varphi_{\alpha}\right\rangle\left\langle\varphi_{\alpha} \mid \xi\right\rangle\left\langle\xi \mid \varphi_{\beta}\right\rangle\left\langle\varphi_{\beta} \mid \varphi_{p}\right\rangle \\
& \left\langle\varphi_{q} \mid \varphi_{\mu}\right\rangle\left\langle\varphi_{\mu} \mid \varsigma\right\rangle\left\langle\varsigma \mid \varphi_{\nu}\right\rangle\left\langle\varphi_{\nu} \mid \varphi_{n}\right\rangle \\
& \rho_{p q}(0) e^{-B^{2}(t)\left(\chi_{\xi}-\chi_{\varsigma}\right)^{2} / 4-i C(t)\left(\chi_{\xi}^{2}-\chi_{\varsigma}^{2}\right)}
\end{aligned}
$$

Here,

$$
\begin{aligned}
B^{2}(t) & =8 \sum_{k} \frac{\left|g_{k}\right|^{2}}{\omega_{k}^{2}} \sin ^{2} \frac{\omega_{k} t}{2} \operatorname{coth} \frac{\beta \omega_{k}}{2}, \\
C(t) & =\sum_{k} \frac{\left|g_{k}\right|^{2}}{\omega_{k}^{2}}\left(\omega_{k} t-\sin \omega_{k} t\right),
\end{aligned}
$$

and $|\xi\rangle,|\varsigma\rangle \in\{|0\rangle,|1\rangle\}$. $B^{2}(t)$ will affect the decoherence but $C(t)$ will not it quantifies purely a shift of the energy levels of the qubit system. When the summation in Eq. (27) is converted to integration in the limit of infinite number of the bath modes, one has

$$
B^{2}(t)=8 \int d \omega W(\omega) g(\omega)^{2} \omega^{-2} \sin ^{2} \frac{\omega t}{2} \operatorname{coth} \frac{\beta \omega}{2},
$$

for the real $g(\omega)$. By using of Eq. (11) we can yield a good qualitative estimate of the relaxation behavior [7], [17. From Ref. 13] we know the condition of Eq. (9) is $\omega_{c} \hbar \gg E_{J}$ and $\omega_{c} \hbar \gg k_{B} T$. It is big enough for $\omega_{c}$ to take $\omega_{c} \hbar=200 \mu e v$. Thus, in the following numerical simulations we set the cutoff frequency $\omega_{c}=200$ (the unit is the reciprocal of $t^{\prime} s$ ). Evaluating Eq.(26) we can obtain the evolution of the density matrix elements of the deduce density matrix $\rho$ as

$$
\begin{aligned}
& \rho_{10}(t)=\frac{1}{2} \rho_{10}\left(1-e^{-B^{2}(t)}+e^{i t E_{J}}+e^{i t E_{J}-B^{2}(t)}\right), \\
& \left.\rho_{11}(t)=\frac{1}{2} \rho_{00}\left(1-e^{-B^{2}(t)}\right)+\frac{1}{2} \rho_{11}\left(1+e^{-B^{2}(t)}\right) 29\right)
\end{aligned}
$$

where $\rho_{11}=\rho_{11}(0), \rho_{10}=\rho_{10}(0)$. The evolution of the closed qubit is $\rho_{11}^{i}(t)=\rho_{11}$, and $\rho_{10}^{i}(t)=\rho_{10} e^{i t E_{J}}$. So we have

$$
\begin{aligned}
\sigma_{10}(t) & =\frac{1}{2} \rho_{10}\left(1-e^{-B^{2}(t)}\right)\left(1-e^{i t E_{J}}\right), \\
\sigma_{11}(t) & =\frac{1}{2}\left(1-e^{-B^{2}(t)}\right)\left(\rho_{00}-\rho_{11}\right) .
\end{aligned}
$$


Then, we have

$$
\begin{aligned}
\|\sigma(t)\|_{\lambda}= & \frac{1}{2}\left(1-e^{-B^{2}(t)}\right) \\
& \left\{\left(\rho_{00}-\rho_{11}\right)^{2}+4\left|\rho_{10}\right|^{2} \sin ^{2} \frac{E_{J} t}{2}\right\}^{\frac{1}{2}}
\end{aligned}
$$

The result is similar but not same to the norm in [7] where $H_{s}=-\frac{\Omega}{2} \sigma_{z}$ and $\Lambda_{s}=\sigma_{x}$.

In the following, we numerically analyze the decoherence. In the following calculation, three pure initial states are chosen, they are $|\varphi\rangle_{0}=(1,0)^{T}$, (corresponding to point); $|\varphi\rangle_{1}=\left(\frac{\sqrt{3}}{2}, \frac{1}{2}\right)^{T}$, (corresponding to above line); $|\varphi\rangle_{2}=\left(\sqrt{\frac{1}{2}}, \sqrt{\frac{1}{2}}\right)^{T}$, (corresponding to below line), [because it has been shown that evaluation of the supremum over the initial density operators in order to find $D$, see Eq. (15) one can do over only pure-state density operators [7]. We choose $E_{J}=51.8 \mu \mathrm{ev}$ according to [15], and $T=30 \mathrm{mK}$. In the model the typical impedance of the control line is $R \approx 50 \Omega$. Since $R_{Q} \approx 6.5 \mathrm{k} \Omega$, Y. Maklin et al. have suggested a value $\eta \approx 10^{-6}$ for numerical simulations. We plot the norms $\|\sigma\|_{\lambda}$ versus time $t$ in Fig.1.

\section{Fig1.a, Fig1.b}

Fig.1: Norms $\|\sigma\|_{\lambda}$ versus time $t$, where the points and lines correspond to different initial states (see the text), $E_{J}=51.8 \mu \mathrm{ev}, T=30 \mathrm{mk}$ and $\eta=10^{-6}$. The unit of the time in the Figs. is $6.582 \times 10^{-10} \mathrm{~s}$.

It is shown that when the initial state is $\rho(0)=$ $|\varphi\rangle_{00}\langle\varphi|,\|\sigma(t)\|_{\lambda}$ is the maximum and it equals to $D=\frac{1}{2}\left(1-e^{-B^{2}(t)}\right)$ (plotted by points in the Figs.). We denote the low decoherence $\left(D \leq 10^{-4}\right)$ time $\tau^{l d}$. From Fig.1a we obtain $\tau^{l d} \approx 7.5 \times 10^{-2} \times 6.582 \times 10^{-10} s=$ 49.4ps. We denote the elementary gate operation time, the characteristic time $\tau^{g}$. In this case, $\tau^{g}=\hbar / E_{J} \approx$ 12.7ps. It is shown that the low decoherence time is larger than the single gate operation time, namely, $\tau^{l d}>\tau^{g}$. The fact means that within the whole time of elementary gate operation, $D \leq 10^{-4}$. Theoretically, the design can satisfy the DiVincenzo low decoherence criterion. A further study shows that when the temperature decreased and the Josephson energy $E_{J}$ increased the qubit can be improved. It is also shown that the decoherence increase with the increasing of dimensionless strength of the dissipation $\eta$.

\section{CONCLUSIONS}

In this paper we investigated the short-time decoherence of the JCQ. We show schematically the behavior of $\|\sigma(t)\|_{\lambda}$. It is shown that for a JCQ the decoherence derive from the dissipation is small enough according to the DiVincenzo criterion. It has been shown that the decoherence of JCQ derive not only from the dissipation but also from the quantum leakage. Fazio et al. pointed that the decoherence from quantum leakage is also not serious in JCQ model [18. The two aspects information shows that the JCQ may be a good candidate of qubit for quantum computation.

\section{Acknowledgments}

I would like to thank Prof. Vladimir Privman and Dr. Leonid Fedichkin for their helpful discussions. A comment by an anonymous referee has greatly improved the paper. The project was supported by National Natural Science Foundation of China (Grant No. 10347133) and Ningbo Youth Foundation (Grant No. 2004A620003).
[1] D. P. DiVincenzo, "Topics in Quantum Computers", in Mesoscopic Electron Transport, edited by L. Kowenhoven, G. Schon and L. Sohn, NATO ASI Series E, (Kluwer Ac. Publ., Dordrecht, 1997) p.657, cond-mat/9612126

[2] M. Governale, M. Grifoni, and G. Schon, Chem. Phys. 268, 273 (2001).

[3] A. J. Berkley, H. Xu, M. A. Gubrud, R. C. Ramos, J. R. Anderson, C. J. Lobb, and F. C. Wellstood, Phys. Rev. B 68, 060502(R) (2003).

[4] A. G. Redfield, Adv. Magn. Reson. 1, 1 (1965).

[5] Y. Makhlin, G. Schön, and A. Shnirman, Rev. Mod. Phys. 73, 357 (2001).

[6] W. H. Louisell, Quantum Statistical properties of Radiation, Wiley, 1973.
[7] V. Privman, J. Stat. Phys. 110, 957 (2003); Mod. Phys. Lett. B 16, 459 (2002); Proc. SPIE 5115, 345 (2003), cond-mat/0303157

[8] L. Fedichkin, A. Fedorov, V. Privman, Proc. SPIE 5105, 243 (2003), cond-mat/0303158 L. Fedichkin, A. Fedorov, Phys. Rev. A 69, 032311 (2004), quant-ph/0309024

[9] H. Grabert and G.-L. Ingold, Superlattices and Microstructures 25, 915 (1999).

[10] A. Shnirman, G. Schön, and Z. Hermon, Phys. Rev. Lett. 79, 2371 (1997).

[11] M. Goernale, M. Grifoni, and G. Schön, Chem. Phys. 268, 273 (2001).

[12] Y. Maklin, G. Schön, and A. Shnirman, Chem. Phys. 295, 1(2003).

[13] A. J. Leggett, S. Chakravarty, A. T. Dorsey, M. P. A. 
Fisher, A. Garg and W. Zwerger, Rev. Mod. Phys. 59, 1 (1987).

[14] S. K. Gray, and J. M. Verosky, J. Chem. Phys. 99, 8680 (1993).

[15] Y. Nakamura, Y. A. Pashkin, and J. S. Tsai, Nature (London) 398, 786 (1999).
[16] S. Blanes, and P. C. Moan, Phys. Lett. A 265, 35 (2000).

[17] N. G. van Kampen, J. Stat. Phys. 78, 299 (1995).

[18] R. Fazio, G. M. Palma, and J. Siewert, Phys. Rev. Lett. 83, 5385 (1999). 


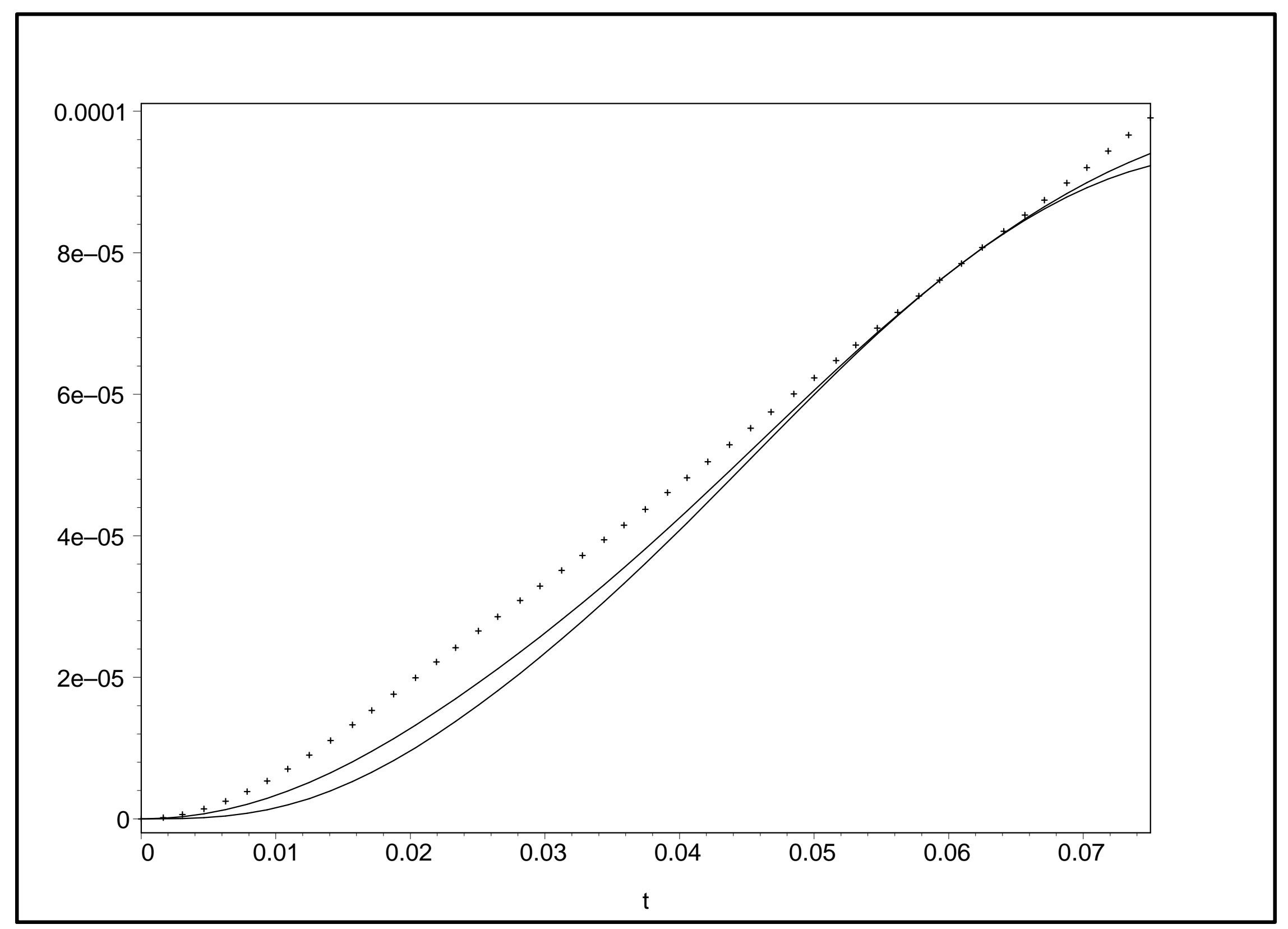




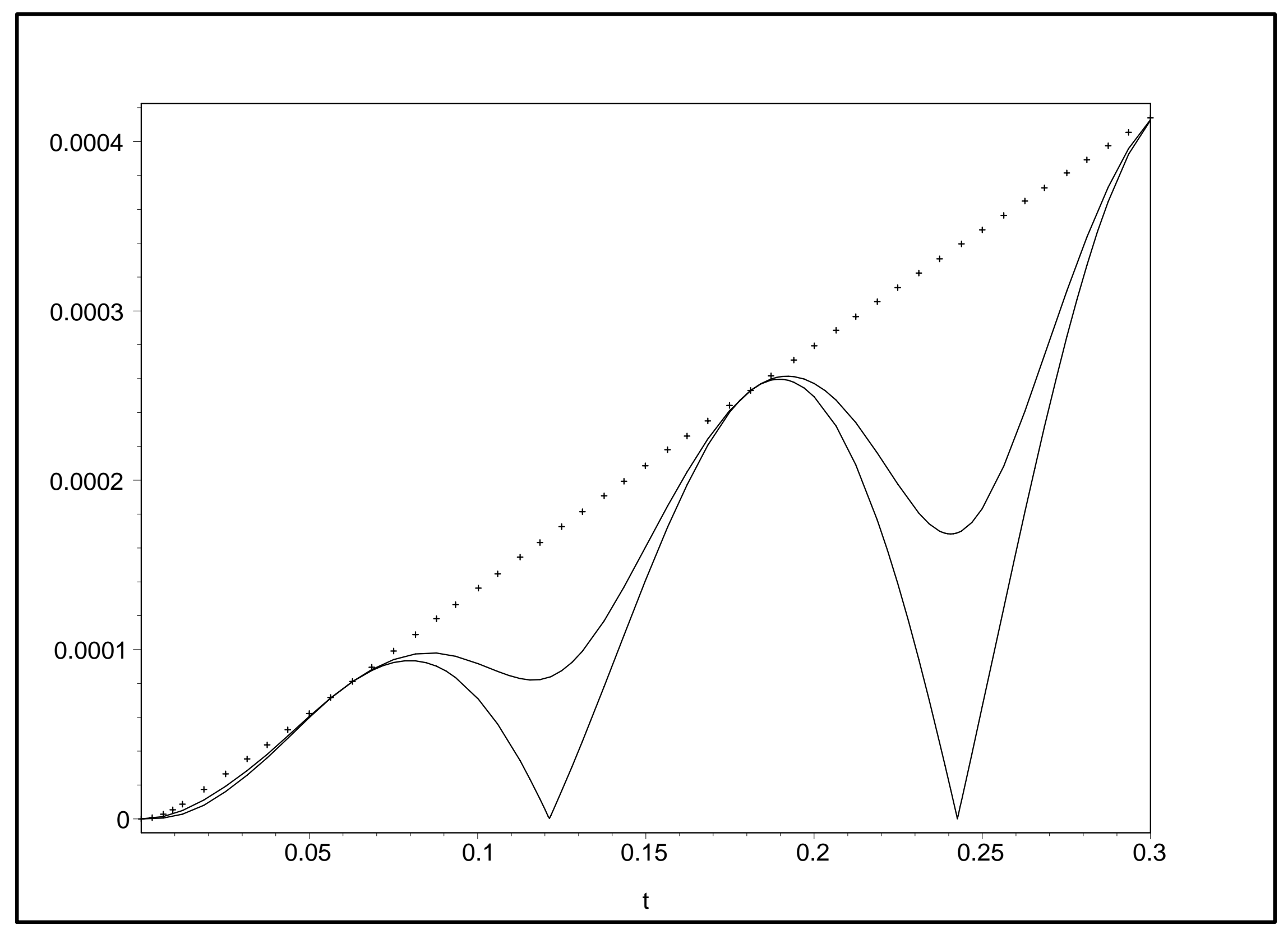

